University of Massachusetts Amherst

ScholarWorks@UMass Amherst

1992

\title{
Closed-Loop Recirculating Manifold for Matrix Isolation in Flow Injection Flame Atomic Absorption Spectrometry. Analysis of Silver Electrolysis Solutions
}

Ebenezer Debrah

University of Massachusetts Amherst

Julian Tyson

University of Massachusetts Amherst

MW Hinds

Follow this and additional works at: https://scholarworks.umass.edu/chem_faculty_pubs

Part of the Chemistry Commons

\section{Recommended Citation}

Debrah, Ebenezer; Tyson, Julian; and Hinds, MW, "Closed-Loop Recirculating Manifold for Matrix Isolation in Flow Injection Flame Atomic Absorption Spectrometry. Analysis of Silver Electrolysis Solutions" (1992). Talanta. 1415.

Retrieved from https://scholarworks.umass.edu/chem_faculty_pubs/1415 


\title{
CLOSED-LOOP RECIRCULATING MANIFOLD FOR MATRIX ISOLATION IN FLOW INJECTION FLAME ATOMIC ABSORPTION SPECTROMETRY. ANALYSIS OF SILVER ELECTROLYSIS SOLUTIONS
}

\author{
Ebenezer Debrah and Julian F. Tyson* \\ Department of Chemistry, University of Massachussets, Amherst, MA 01003, U.S.A. \\ MichaEL W. HindS \\ Royal Canadian Mint, 320 Sussex Drive, Ontario, Ottawa, Canada K1A 0G8
}

(Received 27 March 1992. Accepted 31 March 1992)

\begin{abstract}
Summary-Two flow injection procedures have been investigated for the determination of some elements in silver electrolysis solutions, for which the problem of the formation of silver acetylide needs to be addressed. A single line manifold was found to give acceptable results for limited time periods, but for prolonged operation it was necessary to remove the silver. This was achieved with a recirculating reactor in which the silver was precipitated as the chloride and retained on a filter of nylon fibers. Good recoveries of copper, iron, nickel and zinc from solutions containing up to $100 \mathrm{~g} / \mathrm{l}$. silver were obtained with over $95 \%$ of the silver retained on the filter. The filter was regenerated rapidly by flushing with ammonia solution.
\end{abstract}

A major driving force in research in analytical atomic spectrometry is the need to remove matrix interferences. The use of flow injection (FI) techniques for the separation of analyte and matrix species is proving to be of considerable versatility and several methods of FI separation and preconcentration have recently been reviewed.' Although most of the FI procedures have already been described in an off-line batch mode, there are some practical problems associated with their use which have mitigated against their use in favor of some instrumental based methods of interference removal or compensation. As well as the dangers of contamination and loss, batch procedures are often labor intensive and time consuming. Flow injection methodology, as an approach to automation, is a versatile alternative to manual procedures. Due to the inherent high precision of flow-based procedures, most of the physical and chemical manipulations of the sample can be reduced to the actions of filling and injecting the contents of a sample loop. Flow injection techniques also have the additional benefit of providing a safe, contamination-free enclosed sample handling system.

*Author for correspondence.
Precipitation is the oldest method of chemical separation. ${ }^{2}$ Precipitation methods are satisfactory for macro separations, although precipitates are usually contaminated with foreign ions present in solution, although these would not themselves have been precipitated under the given experimental conditions. Developments in trace analysis and the methods of separation and preconcentration have led to the study of the possibility of using precipitation techniques for matrix isolation. Precipitation is useful for removing the major constituent of a sample if this interferes with the subsequent determination of trace components. Conditions can be chosen such that a considerable amount, if not all, of the major constituent can be precipitated without causing losses, by co-precipitation, of the trace constituents to be determined. For example, Karabash et al..$^{3}$ studied coprecipitation of trace elements with the aid of radio-tracers and have shown that in the precipitation of lead sulfate from a $6 M$ nitric acid solution, many trace elements remained $(85-100 \%)$ in solution, which is sufficient for trace analysis purposes.

Several groups of workers have shown tha it is possible to use precipitation and redissolution in a flow injection manifold for the purposes of preconcentration of trace 
components and the removal of potentially interfering matrix components. For the small quantities of precipitate involved it is possible to retain the solid phase on an in-line filter of either stainless steel, $^{4}$ cellulose acetate $^{5}$ or even to retain the analyte element co-precipitated on the interior of an open knotted tube reactor. ${ }^{6}$

The insertion of a filter directly in-line does not cause a problem when the amount of precipitate is small, but for large amounts of solid the use of a precipitation manifold with direct coupling to an atomic spectrometer presents some difficulties due to the changes in flow rate which occur as the precipitate collects on the filter medium. This is particularly true if a high concentration of a matrix species is to be removed by precipitation.

To overcome this problem, the use of a recirculating, closed-loop manifold for sample pretreatment is described. The use of closed loop recirculating systems in flow systems predates flow injection methodology ${ }^{7}$ and continuous flow recirculation manifolds have been used as part of sample pretreatment procedures. Carbonell et al. ${ }^{8}$ have described the digestion of sewage sludge samples in a continuous flow reactor. The aim of the study was the on-line digestion and the determination of trace metallic elements in solid samples using the slurry approach coupled with microwave oven digestion in an FIA system. In this procedure the partially digested sample was returned into a sample reservoir and a subsample was injected into a single line manifold for transport to the flame atomic absorption spectrometer. Lazaro et al. ${ }^{9}$ described a procedure for the on-line leaching of iron from plant material with the aid of ultrasound and the entire recirculating loop formed the loop of the injection valve. On injection, the sample stream merged with a reagent stream containing 1,10-phenanthroline and the product of the reaction was detected spectrophotometrically at $512 \mathrm{~nm}$. The use of recirculating loops for several different flow injection sample pretreatment procedures has recently been discussed by Tyson et al. ${ }^{10}$ These procedures concerned dilutions for calibration purposes, on-line extraction and matrix isolation.

The determination of metals in solution with high concentrations of silver by flame atomicabsorption spectrometry with an air/acetylene flame can be problematical due to the formation of solid silver acetylide in the spray chamber. This leads to blockage of both the nebulizer and the burner and it is a serious hazard as it may detonate if allowed to dry. ${ }^{11}$

The aim of these studies was to investigate the possibilities for determining trace amounts of metals in silver electrolysis solutions by matrix removal of the silver by precipitation and retention on an on-line filter.

\section{EXPERIMENTAL}

Two atomic absorption spectrometers with continuum source background correction (Perkin-Elmer models 5000 and 1100B) were used. For the model 5000, the signal was recorded on a Houston Instrument Model 200 $\mathrm{XY}$ recorder connected to the analog signal output of the spectrometer. For the model $1100 \mathrm{~B}$, the signal was printed on an Epson model LQ-850 printer. Hollow cathode lamps were operated at the manufacturer's recommended currents. Wavelengths of $324.8 \mathrm{~nm}$ for copper, $232 \mathrm{~nm}$ for nickel, $248.3 \mathrm{~nm}$ for iron, $213.7 \mathrm{~nm}$ for zinc and $328.1 \mathrm{~nm}$ for silver were used and the appropriate single element hollow cathode lamp was used in each case. An airacetylene flame was used in all determinations. The instruments were used for each element according to the manufacturers' specification.

The single line manifold is shown in Fig. 1. A Gilson Minipuls 3 variable speed peristaltic pump was used with a Rheodyne model 5020 6 -way injection valve. Teflon tubing $(0.08 \mathrm{~mm}$ i.d.) tubing and flangeless fittings (Supelco) were used to complete the manifold. The injection loop volume was $70.7 \mu \mathrm{l}$. The recirculating flow manifold (see Fig. 2) was constructed from PTFE tubing of $0.8 \mathrm{~mm}$ or $0.5 \mathrm{~mm}$ internal diameter. The filter column consisted of a borosilicate glass column $50 \mathrm{~mm} \times 3 \mathrm{~mm}$ (Omnifit) filled with nylon fibers (PolyScience Inc., Pennsylvania). A multichannel variable speed peristaltic pump (Ismatec model MS-4 Reglo 8-100, Cole Parmer, Chicago, IL, U.S.A.) was used to pump liquid in the manifold. Valves were either 6-port rotary valves (Rheodyne model 50, Alltech, South College, Pennsylvania) for sample introduction into the manifold and

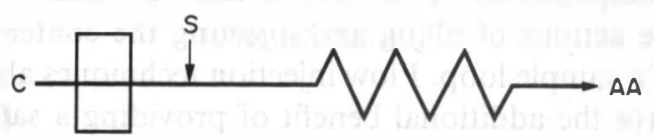

Fig. 1. Single line manifold. $C$ is a distilled water carrier stream, $S$ is the sample injection valve and $A A$ is the model 5000 spectrometer. The connection between the valve and the detector was the minimum length of tubing possible. 


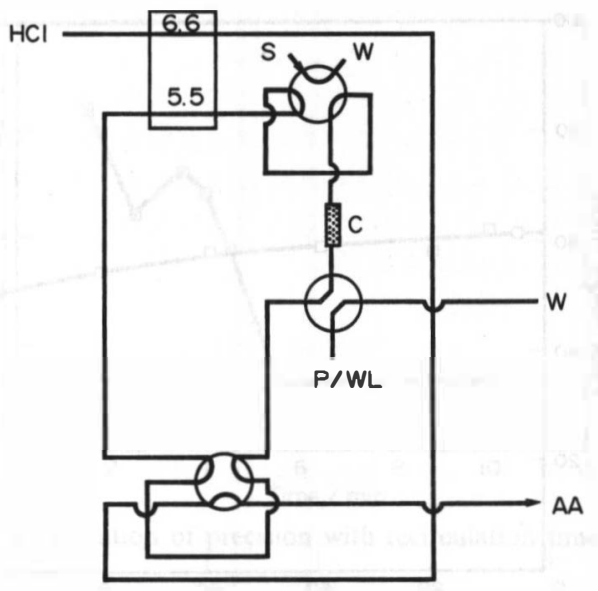

Fig. 2. Recirculation closed loop manifold for the matrix isolation of silver in the determination of trace metals. AA is the model 1100B Spectrometer, $\mathrm{S}$ is the sample injection valve, $\mathrm{P} / \mathrm{WL}$ is the precipitant/wash liquid, $\mathrm{HCl}$ is $2 M$ hydrochloric acid solution and $\mathrm{W}$ is waste.

the injection of subsamples into the spectrometer or 4-port distribution valve (Omnifit, model 1114) for introduction of the precipitant or the wash liquid into the manifold.

The manifold was operated by first filling the loop with the precipitant (hydrochloric acid) via the four way selection valve and then injecting the sample into the manifold at the point labelled $\mathrm{S}$ in Fig. 2 and turning the valve so that the precipitant flowed through the sample loop. The precipitate formed was retained on the filter column labelled $\mathrm{C}$ and the sample was allowed to circulate for a predetermined period of time after which a subsample of the contents in the recirculation loop was injected into the spectrometer via a single line manifold. After injection of the subsample, the loop was filled via the selection valve with ammonium hydroxide solution $(1+1)$ to dissolve precipitate on the column and wash the loop. The loop was then rinsed with distilled demineralized water and was then filled with fresh precipitant and the procedure repeated.

Copper, iron, nickel and zinc standards were made from the dilution of $1000 \mathrm{mg} / \mathrm{l}$. standards (Fisher Scientific). The silver solutions were prepared from silver nitrate crystals (Fisher Scientific). Hydrochloric acid (Baker Instraanalyzed, Fisher Scientific) was used for the precipitation reactions and ammonium hydroxide (ACS Certified, Fisher Scientific) was used for all washings. Distilled dimineralized water (Barnstead Inc.) was used for all dilutions and washing of the manifold. All solutions were stored in high density polyethylene bottles.

\section{Procedures}

Single line manifold. The effect of flow and the concentration of dissolved silver on copper absorbance were investigated. To investigate the effect of the formation and build-up of precipitate in the spray chamber a 2.0 -ppm copper solution was introduced continuously at a fixed flow rate of $2.5 \mathrm{ml} / \mathrm{min}$ between each change in silver concentration. The calibration solutions covered the range 0-60 ppm copper. Two samples were analyzed by the single line flow injection procedure and by EDTA titration.

\section{Recirculating loop manifold}

Effect of the recirculation time. This was studied by filling the loop with demineralized water and injecting samples of $10 \mathrm{mg} / 1 . \mathrm{Cu}$ and allowing them to recirculate for different lengths of time between 1.5 and $10 \mathrm{~min}$.

Effect of the concentration of the precipitant. The effect of the concentration of the $\mathrm{HCl}$ precipitant over the range 0.2 to $2.0 \mathrm{M}$ was studied for a solution containing $10 \mathrm{mg} / \mathrm{l} . \mathrm{Cu}$ and $50 \mathrm{~g} / \mathrm{l}$. Ag.

Tolerable level of silver in the sample injected. The effect of varying the silver concentration between 1 and $100 \mathrm{~g} / 1$. was studied for a fixed concentration of copper.

Optimization of the flow parameters. The flow rate of the carrier to the spectrometer was optimized by injecting a subsample of the solution into the spectrometer at varying flow rates. The flow rate was varied between 3.2 and $8.6 \mathrm{ml} / \mathrm{min}$. The flow rate within the loop was also varied between the same values.

Effect of the sample volume injected. The volume of sample solution injected into the manifold was varied between 70 and $185 \mu \mathrm{l}$.

Effect of subsample injected into the spectrometer. Volumes of the subsample injected into the carrier stream for transport to the spectrometer were varied between 111 and $462 \mu 1$.

Recovery studies. The recovery of copper, zinc, nickel and iron at concentrations of 10 $\mathrm{mg} / \mathrm{l}$. in the presence of varying amounts of silver between 1 and $100 \mathrm{~g} / \mathrm{l}$. were studied.

Breakthrough of silver. The amount of silver that was not retained on the filter column was investigated by monitoring the silver absorbance for the same samples used for the recovery studies.

Calibration. Under the optimum conditions, a $163-\mu 1$ sample volume and a subsample of 


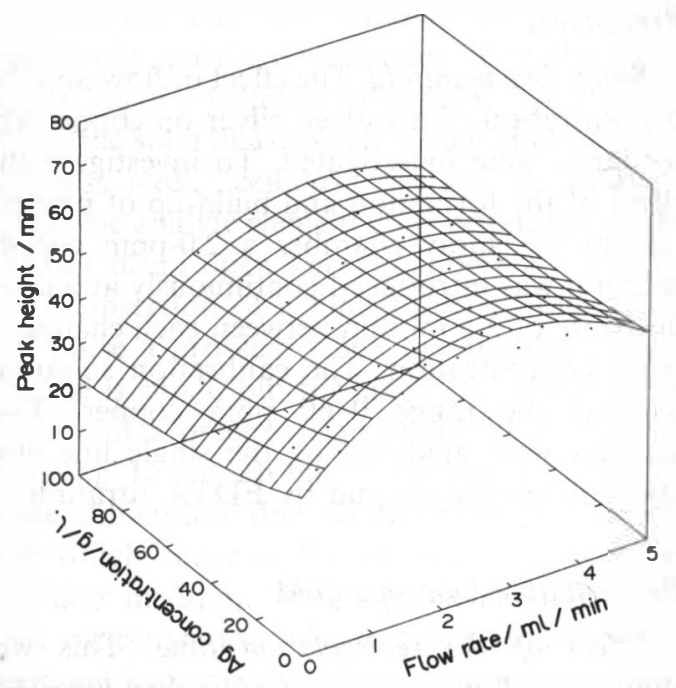

Fig. 3. Response surface for variation of flow rate and silver concentration for the single line manifold.

$237 \mu 1$, calibration plots were generated for copper, nickel, iron and zinc in the range of 0 to $30 \mathrm{mg} / \mathrm{l}$. and the amounts of these trace elements in synthetic samples with silver matrix determined. Three samples from the Royal Canadian Mint (RCM 1, RCM 2 and RCM 3) were also analyzed for copper by the standard addition method. Both the normal calibration plots and the standard additions plots were generated using standard solutions containing $1000 \mathrm{mg} / \mathrm{l}$. silver.

\section{RESULTS AND DISCUSSION}

\section{Single line manifold}

The effects of the flow rate and silver concentration are shown in Fig. 3. The surface plot shows the variation of the peak height with changes in both flow rate and Ag concentrations. Peak height remained independent of the silver concentration except at very high concentrations. Changes in flow rate affected the measured absorbance. A decrease in the flow

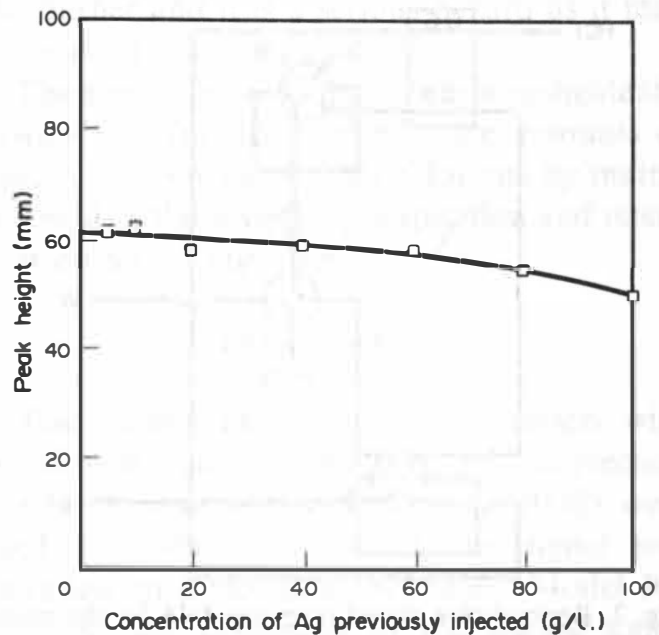

Fig. 4. Response of a constant concentration of copper as a function of the concentration of silver injected.

rate below $2.5 \mathrm{ml} / \mathrm{min}$ caused a significant decrease in the peak heights observed. The effect of the build up of precipitate is shown in Fig. 4. There was a small change in response over the silver concentration range indicating that the amount of this type of material deposited in the nebulizer does not seriously impede the solution from reaching the flame in the short term. However, over prolonged periods of operation it was found necessary to clean the system.

A typical analysis sequence is shown in Fig. 5. The values for the copper concentrations of two samples determined by this procedure were 0.44 and $5.13 \mathrm{~g} / \mathrm{l}$. whereas the values obtained by EDTA titration were 0.441 and $5.36 \mathrm{~g} / \mathrm{l}$., respectively.

\section{Recirculating loop manifold}

Peak height was used to characterize the absorbances. All the standard deviations and relative standard deviations quoted are based on four replicate measurements. The total volume of the recirculation loop was $660 \mu$ l, the optimum volume of sample injected in the manifold

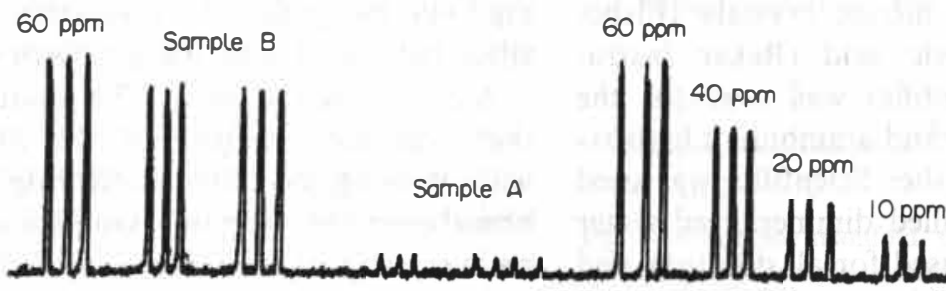

Fig. 5. Recorder trace for single line manifold for the determination of copper at $222.6 \mathrm{~nm}$ in diluted 


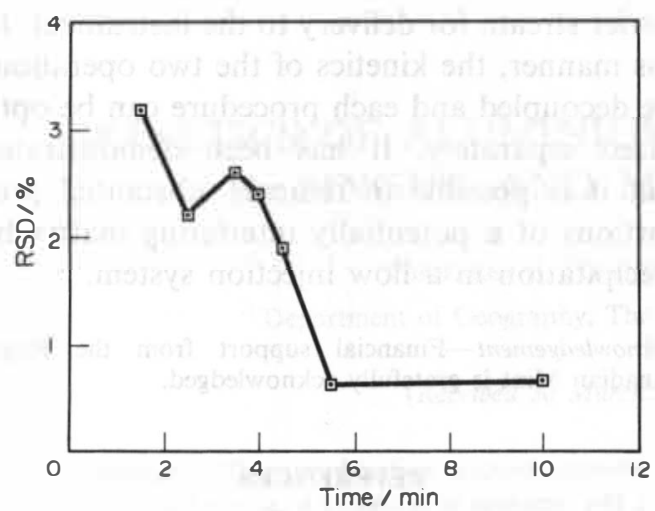

Fig. 6. Variation of precision with recirculation time.

was $163 \mu l$ and the optimum subsample volume injected into the spectrometer was $237 \mu 1$. It was observed that circulating the sample in the manifold for $4.5 \mathrm{~min}$ was sufficient to let the contents of the sample mix so that a reasonable reproducibility could be obtained, see Fig. 6. For times of less than $4.5 \mathrm{~min}$ the reproducibility was poor. In effecting a good filtration for these studies the differences between the two principal types of colloidal precipitates, hydrophobic and hydrophilic, is important. Hydrophilic precipitates are difficult to separate by filtration and to wash and are likely to coprecipitate numerous substances. On the other hand, hydrophobic precipitates, such as silver chloride, have a low water affinity and are purer and more readily filterable. ${ }^{2}$ It was observed that the precipitation was very efficient at a hydrochloric acid concentration of $2 M$ and this was chosen for further experiments. This concentration was high enough such that only a small amount of precipitate was not retained on the first pass of the precipitate through the filter column. The recoveries obtained are shown in Table 1. These results indicate there is no problem with coprecipitation and also varying silver concentrations do not give significantly different recoveries. The recoveries for nickel were higher than for the other elements and to investigate a possible enhancement by silver or iron, solutions containing varying amounts silver or iron in a fixed concentration of nickel were nebulized directly into the spectrometer. However, no
Table 2. Calibration plots

$r^{2}$

\begin{tabular}{ll}
\hline Nickel & \\
Absorbance $=1.3500 \times 10^{-3}+3.4510 \times 10^{-3}[\mathrm{Ni}]$ & 0.998 \\
Copper & \\
Absorbance $=4.9493 \times 10^{-3}+4.0174 \times 10^{-3}[\mathrm{Cu}]$ & 0.995 \\
Zinc & \\
Absorbance $=1.8911 \times 10^{-2}+1.6589 \times 10^{-2}[\mathrm{Zn}]$ & 0.991 \\
Iron & \\
Absorbance $=1.8893 \times 10^{-3}+4.5314 \times 10^{-3}[\mathrm{Fe}]$ & 0.996 \\
\hline
\end{tabular}

significant difference between the absorbance of the sample containing no silver and the samples containing the largest amount of silver (1000 $\mathrm{mg} / \mathrm{l}$.) or iron $(500 \mathrm{mg} / \mathrm{l}$.) was observed.

There was no significant difference between the peak heights obtained for the different flow rates in the recirculating loop. A flow rate of 5.5 $\mathrm{ml} / \mathrm{min}$ was chosen for further experiments. It was observed that large volumes of samples did not significantly affect the peak height, although peak area increased proportionately. In a manner similar to that observed with the single line manifold, as the flow rate of the carrier was varied it was observed that the peak height increased in a direct proportion. The highest flow rate used, $8.6 \mathrm{ml} / \mathrm{min}$, gave the highest mean absorbance but the precision was poor so a flow rate of $6.6 \mathrm{ml} / \mathrm{min}$ was used throughout the experiment.

The manifold can accommodate a silver concentration of up to $90 \mathrm{~g} / \mathrm{l}$. but above this concentration the flow in the manifold is impeded and the precision of the measurements get worse. For example the RSD for the recovery of copper in the presence of $100 \mathrm{~g} / \mathrm{l}$. silver was $15 \%$ compared with $4 \%$ in the presence of $90 \mathrm{~g} / \mathrm{l}$. silver. The precision (measured as the relative standard deviation of 4 replicate determinations) was below 5\% for almost all the measurements made at or below this concentration of silver. It was observed that even for a sample containing $90 \mathrm{~g} / \mathrm{l}$. silver, more than $95 \%$ of the silver was retained on the column. It was noted that proportion of the precipitate retained increased as the silver content in the solution analyzed increased. This was

Table 1. Recoveries (\%) of metals from a silver matrix

\begin{tabular}{lccccc}
\hline Element & $50 \mathrm{~g} / \mathrm{l} . \mathbf{A g}$ & $60 \mathrm{~g} / \mathrm{l} . \mathbf{A g}$ & $70 \mathrm{~g} / \mathrm{l} . \mathbf{A g}$ & $80 \mathrm{~g} / \mathrm{l} . \mathbf{A g}$ & $100 \mathrm{~g} / 1 . \mathbf{A g}$ \\
\hline $\mathrm{Zn}$ & 112.0 & - & 114.0 & - & - \\
$\mathrm{Cu}$ & 97.2 & - & 106.5 & - & 95.0 \\
$\mathrm{Fe}$ & 99.8 & - & 96.5 & - & - \\
$\mathrm{Ni}$ & 101.4 & 129.0 & - & 129.0 & - \\
\hline
\end{tabular}


Table 3. Determination of trace elements in synthetic samples

\begin{tabular}{lccc}
\hline & $\begin{array}{c}\text { Added } \\
(\mathrm{mg} / \mathrm{l} .)\end{array}$ & $\begin{array}{c}\text { Found } \\
(\mathrm{mg} / \mathrm{l} .)^{*}\end{array}$ & RSD (\%) \\
\hline Iron sample 1 & 18 & $18.67 \pm 0.74$ & 4.0 \\
Iron sample 2 & 12 & $12.23 \pm 0.35$ & 2.9 \\
Zinc sample 2 & 15 & $16.53 \pm 0.24$ & 1.5 \\
Copper sample 1 & 15 & $16.53 \pm 0.24$ & 1.5 \\
Copper sample 2 & 12 & $11.34 \pm 0.30$ & 2.6 \\
Nickel sample 1 & 15 & $14.60 \pm 0.35$ & 2.4 \\
Nickel sample 2 & 18 & $8.11 \pm 1.77$ & 9.8 \\
\hline
\end{tabular}

* \pm terms are standard deviation based on 4 replicate measurements.

considered to be related to an increased rate of crystal growth at higher concentrations. The calibration plots for all the elements were linear (see Table 2) and the results obtained for the synthetic samples are shown in Table 3 . Two samples previously analyzed by the Royal Canadian Mint and found to have 3.65 and $3.00 \mathrm{~g} / \mathrm{l}$. copper were analyzed and found to contain $3.72 \pm 0.12$ and $3.10 \pm 0.12 \mathrm{~g} / \mathrm{l}$. copper, respectively. The \pm terms are standard deviations calculated from the calibration plots. $^{12}$

\section{CONCLUSION}

The coupling of a chemical pretreatment whose kinetic character would make interfacing a flow injection manifold directly with a flame atomic absorption spectrometer difficult has been achieved by the use of a flow injection valve as interface between the reactor and the carrier stream for delivery to the instrument. In this manner, the kinetics of the two operations are decoupled and each procedure can be optimized separately. It has been demonstrated that it is possible to remove substantial proportions of a potentially interfering matrix by precipitation in a flow injection system.

Acknowledgement-Financial support from the Royal Canadian Mint is gratefully acknowledged.

\section{REFERENCES}

1. J. F. Tyson, Spectrochimica Acta Rev., 1991, 14, 169.

2. J. Minczewski, J. Chwastowska and R. Dybczynski, Separation and Preconcentration Methods in Inorganic Trace Analysis, Ellis Horwood, Chichester, 1982.

3. A. G. Karabash, L. S. Bondarenko, G. G. Morozova and Sh. I. Peizulaev, Zh. Analit, Khim., 1960, 15, 623.

4. P. Martinez-Himinez, M. Gallego and M. Valcarcel, Analyst, 1987, 112, 1233.

5. E. Debrah, C. E. Adeeyinwo, S. R. Bysouth and J. F. Tyson, ibid., 1990, 115, 1543.

6. Z. Fang, M. Sperling and B. Welz, J. Anal. At. Spectrom., 1991, 6, 301.

7. H. U. Bergmeyer and A. Hagen, Z. Anal. Chem., 1972, 261, 333.

8. V. Carbonell, M. de la Guardia, A. Salvador, J. L. Burguera and M. Burguera, Anal. Chim. Acta, 1990, 238, 417.

9. F. Lazaro, M. D. Luque de Castro and M. Valcarcel, ibid., 1991, 242, 283.

10. J. F. Tyson, S. R. Bysouth, E. Grzeszczyk and E. Debrah, Anal. Chim. Acta, 1992, 261, 75.

11. G. D. Muir (ed.), Hazards in the Chemical Laboratory, The Chemical Society, London, 1977.

12. J. C. Miller and J. N. Miller, Statistics for Analytical Chemistry, Ellis Horwood, Chichester, 1984. 